\title{
Magnetic Rayleigh-Taylor instability for Pulsar Wind Nebulae in expanding Supernova Remnants
}

\author{
N. Bucciantini ${ }^{1,2}$, E. Amato ${ }^{3}$, R. Bandiera ${ }^{3}$, J. M. Blondin ${ }^{2}$, and L. Del Zanna ${ }^{1}$ \\ 1 Dip. di Astronomia e Scienza dello Spazio, Università di Firenze, Largo E. Fermi 2, 50125 Firenze, Italy \\ e-mail: niccolo@arcetri.astro.it \\ 2 Department of Physics, North Carolina State University, Raleigh, NC 27695, USA \\ 3 INAF, Osservatorio Astrofisico di Arcetri, Largo E. Fermi 5, 50125 Firenze, Italy
}

Received 1 March 2004 / Accepted 11 May 2004

\begin{abstract}
We present a numerical investigation of the development of Rayleigh-Taylor instability at the interface between an expanding Pulsar Wind Nebula and its surrounding Supernova Remnant. These systems have long been thought to be naturally subject to this kind of instability, given their expansion behavior and the density jump at the contact discontinuity. High resolution images of the Crab Nebula at optical frequencies show the presence of a complex network of line-emitting filaments protruding inside the synchrotron nebula. These structures are interpreted as the observational evidence that Rayleigh-Taylor instability is in fact at work. The development of this instability in the regime appropriate to describe Supernova Remnant-Pulsar Wind Nebula systems is non-trivial. The conditions at the interface are likely close to the stability threshold, and the inclusion of the nebular magnetic field, which might play an important role in stabilizing the system, is essential to the modeling. If Rayleigh-Taylor features can grow efficiently a mixing layer in the outer portion of the nebula might form where most of the supernova material is confined. When a magnetic field close to equipartition is included we find that the interface is stable, and that even a weaker magnetic field affects substantially the growth and shape of the fingers.
\end{abstract}

Key words. instabilities - magnetohydrodynamics (MHD) - stars: pulsars: general - stars: winds, outflows ISM: supernova remnants

\section{Introduction}

Pulsars are rapidly rotating magnetized neutron stars that usually form as the result of a Supernova (SN) explosion. As a consequence of the electromagnetic torques acting on it, a pulsar releases most of its rotational energy in the form of a relativistic magnetized wind. The wind is usually thought to be made of electron-positron pairs and to carry a magnetic field that far enough from the light cylinder is almost purely toroidal (Michel \& Li 1999; Goldreich \& Julian 1969). This outflow is highly relativistic, with a terminal Lorentz factor in the range $10^{4}-10^{7}$. Its confinement by the surrounding Supernova Remnant (SNR) generates a nebula of relativistically hot material that shines through synchrotron and Inverse Compton emission from radio wavelengths up to $\gamma$-rays: this is what we call a pulsar wind nebula (PWN) or "plerion".

During a SN explosion as much as $10^{51}$ erg of energy are released in the form of a blast wave that produces a strong shock propagating in the surrounding medium. The ejected material is initially heated by the blast wave and set into motion. As the ejecta expand, their thermal pressure finally becomes so low as to be dynamically unimportant: from this moment on the expansion can be approximated as homologous (Chevalier \& Soker 1989; Matzner \& McKee 1999). This phase is referred to as "free expansion" of the ejecta.
The evolution of the PWN inside the free expanding ejecta depends on many different parameters such as the pulsar luminosity, the flux anisotropies of the pulsar wind (Komissarov \& Lyubarsky 2003; Del Zanna et al. 2004), the density and velocity distribution in the ejecta (Dwarkadas \& Chevalier 1998; Featherstone et al. 2001; Blondin et al. 1996), as well as the presence of large and/or small scale anisotropies (Chevalier \& Soker 1989; Campbell et al. 2003). As a consequence, the detailed modeling of a single PWN-SNR system requires the knowledge of a number of parameters depending on the specific conditions, that are usually unknown.

The simplest approximation one can make for the time evolution of the PWN size is obtained assuming constant pulsar luminosity and spherical symmetry (Chevalier \& Fransson 1992; van der Swaluw et al. 2001; Bucciantini et al. 2003, 2004). If a radial power law density profile, $\rho \propto r^{-\alpha} t^{\alpha-3}$, is further assumed for the SN ejecta, then the PWN size evolves as $t^{(6-\alpha) /(5-\alpha)}$. For a more detailed description of the various phases of the PWN-SNR evolution see Bucciantini et al. (2003) and references therein.

The interface between the synchrotron nebula and the swept up shell of ejecta has been thought to be RayleighTaylor (hereafter RT) unstable (Chevalier \& Gull 1975; Bandiera et al. 1983). In the case of the Crab Nebula the 
RT instability is expected to be at the origin of the complex network of emission-line filaments protruding into the PWN (Hester et al. 1996, H96 hereafter). The recent images by H96 show that these "radial" filaments are joined at their basis by faint, thin, "tangential", and often somewhat arcuate features, which are interpreted as tracing the location of the RT unstable interface. The filamentary structure presents a clear hierarchy as expected from a multimode instability.

Simulations of the RT instability in the first phase of the PWN-SNR evolution have been presented by Jun in a classical hydrodynamical (HD) regime (Jun 1998, hereafter J98). However, as H96 pointed out, the standard model for PWNe (Kennel \& Coroniti 1984) leads to believe that many of them are magnetically dominated at the contact discontinuity with the SNR. In addition, within the standard framework, the nebular magnetic field, usually expected to be purely toroidal, is tangential to the contact discontinuity. A parallel magnetic field is expected to have a stabilizing effect and possibly even to suppress the formation of fingers (Chandrasekhar 1961; Wang \& Nepveu 1983; Jun et al. 1995). In the recent work by $\mathrm{H} 96$ a comparison with results from classical magnetohydrodynamical (MHD) simulations of the RT instability (Jun et al. 1995) was carried out. The results suggest that, in the case of the Crab Nebula, the magnetic field should be close to the critical value for stability. However, the conditions at the contact discontinuity between PWNe and SNRs are quite different from those adopted both in the formulation of the standard theory (Chandrasekhar 1961) and in the existent MHD simulations (Jun et al. 1995), as we will discuss in the following.

In this paper we present a study of the RT instability in the presence of a tangential magnetic field in the context of PWN-SNR systems. Our analysis is carried out by means of 2D special relativistic MHD (RMHD) simulations. In Sect. 2 we review the standard theory for plane-parallel RT instability, discussing how the main results are modified in the situation under investigation. In Sect. 3 the numerical method and the initial conditions for the simulations are described. Section 4 is dedicated to the numerical results both in the HD and MHD regimes. In Sect. 5 we finally summarize our conclusions.

\section{The Rayleigh-Taylor instability}

RT instability occurs when a heavy fluid is supported by a lighter fluid in a gravitational field, or, equivalently, when a heavy fluid is accelerated by a lighter fluid. This instability can be at work in many different astrophysical contests ranging from supernova explosions (Fryxell et al. 1991) to shock wave interaction with ISM clouds (Stone \& Norman 1992), from accretion onto compact objects (Wang \& Nepveu 1983) to SNR evolution (Chevalier et al. 1992).

In the linear regime, short wavelengths grow faster than long wavelengths. When the system enters the non linear phase the fluid interface assumes its characteristic "mushroomfinger" structure, with the finger penetrating the lighter fluid. As RT fingers grow, the shear between the two fluids gives rise to secondary Kelvin-Helmholtz (KH hereafter) instabilities, which concentrate vorticity in a mushroom cap at the tip of the fingers. The cap increases the drag on the finger slowing down its growth. In the fully non linear phase the evolution of the instability is affected by effects such as mergers and breakup of the fingers, which form a mixing layer between the two fluids.

The evolution of the RT instability is influenced by many different factors. Viscosity tends to reduce the growth rate and to stabilize the system (Plesset \& Whipple 1974). Compressibility (i.e. radiative cooling) can produce thinner and longer fingers (Gardner et al. 1988). However, the most important effects in the astrophysical context are probably those due to the presence of a magnetic field. In general the magnetic field will have both a normal and a tangential component to the interface. We will deal only with the effect of a tangential magnetic field. In fact, pulsar winds, and, as a consequence, PWNe (at least to first approximation) are expected to contain a purely toroidal magnetic field. We will here neglect global instabilities that can remove the axial symmetry of the system, like kink instability (Begelman 1998), and generate a non-negligible magnetic field component perpendicular to the interface. The RT instability at the contact discontinuity with the SNR can in principle generate a radial component as magnetic field lines are forced to bend along the finger.

Let us recall the results of the linear theory for the simple plane parallel case with a uniform tangential magnetic field in both fluids, under the assumption of incompressibility. This situation was first studied analytically by Chandrasekhar (1961). The linear stability theory shows that a tangential magnetic field slows the growth of the RT instability. The growth rate for modes with wave number $k$ parallel to the magnetic field lines is given by:

$w^{2}=\frac{g k\left(\rho_{2}-\rho_{1}\right)-B^{2} k^{2} / 2 \pi}{\left(\rho_{2}+\rho_{1}\right)}$,

where $\rho_{1}$ and $\rho_{2}$ are the densities of the light and heavy fluid respectively, $B$ is the magnetic field, $g$ is the gravitational acceleration (or equivalently the acceleration of the system) and the real part of $w$ is the growth rate.

From Eq. (1) one derives the critical strength the tangential magnetic field should have to stabilize the system against perturbations of wavelength $\lambda$ or smaller:

$B_{\mathrm{c}}=\sqrt{g \lambda\left(\rho_{2}-\rho_{1}\right)}$.

Similarly, the critical wavelength for a given magnetic field is defined as:

$\lambda_{\mathrm{c}}=\frac{B^{2}}{g\left(\rho_{2}-\rho_{1}\right)} ;$

the instability will be suppressed for smaller scales.

In the work by H96 these equations were used to interpret the filamentary network observed in the Crab Nebula as the result of the RT instability in PWN-SNR systems. However, many of the assumptions of the plane parallel theory do not apply in the context of PWNe: the lighter fluid is relativistically hot, the density in the swept-up shell is not uniform, nor is the magnetic field (which is actually relevant only on one side of the interface, the PWN's), all quantities evolve in time so that 
no fixed background conditions can be assumed, and, finally, spherical geometry must be used.

Concerning the relativistic corrections, Allen \& Hughes (1984) have shown that in the HD case the growth rate is still given by Eq. (1) substituting the energy density with the enthalpy. The latter is, for a relativistic magnetized fluid:

$h=\rho c^{2}+\frac{\Gamma}{\Gamma-1} P+\frac{B^{2}}{4 \pi}$,

with $\rho$ the rest mass density, $P$ the thermal pressure, $B$ the magnetic field, and $\Gamma=4 / 3$ the adiabatic coefficient.

Corrections to the incompressible approximation (for modes parallel to the magnetic field), in the case of a strong magnetic field, have been presented so far only to the first order in $B^{2} / P$ by Shivamoggi (1982), who showed that Eq. (1) holds but the denominator and the term in $B^{2}$ have to be multiplied by a factor $1+B^{2} /(8 \pi \Gamma P)$. The second order effect of a strong magnetic field appears to be that of further reducing the growth rate and increasing the stability of the system. Effects due to magnetic pressure are probably the reason why Wang $\&$ Nepveu (1983) in simulations of accretion onto compact objects with high magnetic fields found that (with respect to the standard stability value): "considerably weaker fields are sufficient to stop the infall".

\subsection{Stability criterion and growth rate in the framework of the self-similar evolution}

The equations above are only valid in the plane parallel context, in which uniform density and magnetic field are assumed on both sides of the contact discontinuity and a constant acceleration is considered. However, the interface between the synchrotron nebula and the shell of ejecta is far from this condition. No analytic theory is available for the specific conditions and evolution of the interface. Despite this, we will try to derive from the standard theory above information that will help us in the interpretation of our numerical results. We will use the plane parallel criterion (Eq. (1)), with values of the various quantities derived from the self similar evolution (Chevalier \& Fransson 1992; Bucciantini et al. 2004).

The first thing to notice is that the above equations can be easily simplified in the case one of the two fluids has a much higher density (or enthalpy in the relativistic regime) than the other. This is exactly the case in PWN-SNR systems. For the shell of ejecta (a non relativistic gas) we can simply consider the density, while for the PWN we only need to consider the total pressure.

As shown in previous papers (Bucciantini et al. 2003; Bucciantini et al. 2004), in the framework of the self similar model, the total pressure at the contact discontinuity is independent of the wind (or nebular) magnetization, so that its value and evolution can be derived from a simplified HD description. It turns out that the shell density is about 3-4 orders of magnitude greater that the PWN enthalpy. Therefore the latter can be neglected, and Eqs. (1)-(3) simplified.

Assuming a constant pulsar luminosity and ejecta having a density profile $\rho_{\mathrm{ej}} \propto r^{-\alpha} t^{\alpha-3}$, the PWN expands as $R_{\mathrm{pwn}} \propto t^{\beta}$, with $\beta=(6-\alpha) /(5-\alpha)$. The total pressure of the PWN at the contact discontinuity is given by:

$P_{\mathrm{tot}}(t)=\left[\frac{\beta(\beta-1)}{3-\alpha}+(\beta-1)^{2}\right] \rho_{\mathrm{ej}}\left(R_{\mathrm{pwn}}, t\right)\left(\frac{R_{\mathrm{pwn}}}{t}\right)^{2}$

In principle, the magnetic pressure at the boundary can change in time as the velocity of the contact discontinuity increases. These variations, anyway, will be more important in the early phases, and, in general, we might assume, to a good approximation, that the magnetic pressure is a constant fraction $C$ of the total pressure ( $C \simeq 0.5$ at equipartition).

Concerning the radial variations of the enthalpy and magnetic field in the PWN, we notice that their length-scales are of the same order of the nebular radius, so that using the values at the discontinuity itself would not be too bad an approximation. On the contrary, the swept up shell of ejecta shows a strong gradient of density (J98). To remain within our simplified approach, we will assume for the shell a uniform density, equal to the average:

$\rho_{\mathrm{sh}}\left(R_{\mathrm{pwn}}, t\right)=\frac{\rho_{\mathrm{ej}}\left(R_{\mathrm{pwn}}, t\right)}{(3-\alpha) \Delta}$,

where $\Delta$ is the ratio between the thickness of the shell and the nebular radius. The self similar theory gives a shell thickness corresponding to $\Delta \approx 0.02-0.04$ for values of $\alpha$ ranging from 0 to 2 (J98). These values of $\Delta$ refer to the adiabatic case, while smaller values are found if radiative cooling is considered.

Another important parameter is the acceleration of the system. The effective acceleration of the contact discontinuity can be easily derived in the self similar model and it reads:

$g=\beta(\beta-1)\left(\frac{R_{\mathrm{pwn}}}{t^{2}}\right)$

To preserve the overall self-similarity we will deal with constant angular sizes of the perturbations. The linear wavelength of the perturbation, $\lambda$, will increase in time as the nebula expands:

$\lambda=\theta R_{\mathrm{pwn}}$,

with $\theta=$ const. the angular size. We define the value of the critical density from Eq. (2) as:

$\rho_{\mathrm{c}}=\frac{B^{2}}{g \lambda}$

the system is unstable for $\rho_{\mathrm{sh}}>\rho_{\mathrm{c}}$. In the plane parallel theory the ratio between the shell and critical density would determine the stability properties of the system, as a function of the size of the initial perturbation. For limitations on the validity of this approach depending on the values of $\lambda$ see Sect. 2.2. We will follow the same approach to derive a stability criterion and to determine which parameters of the PWN-SNR system are important in this regard. After some algebra we find from Eqs. (5)-(9):

$\frac{\rho_{\mathrm{sh}}}{\rho_{\mathrm{c}}} \simeq \frac{1}{C} \frac{1}{8 \pi \Delta} \frac{1}{1+(3-\alpha)(\beta-1) / \beta} \theta$. 
In the range $\alpha=0-2$, assuming equipartition $(C \sim 0.5)$ and no cooling, we find that this ratio exceeds unity only for perturbations with $\theta \gtrsim \pi / 10$.

Let us discuss in more detail Eq. (10). The first point to notice is that the definition of the stability criterion does not have any time dependence. This is a direct consequence of the selfsimilarity assumed for the evolution: in this case, perturbations can be stable or unstable, but no transition between these two regimes is allowed for a given angular size. If self-similarity is a good approximation for the evolution, the stability of the interface is independent on the specific age of the PWN-SNR system. Thus numerical results, that in principle refer to a specific moment of the nebular history, can be generalized. In general, the self-similar evolution of PWNe is determined by three dimensional parameters: the pulsar luminosity and the energy and mass ejected during the SN. Nonetheless, none of these quantities appears in Eq. (10), where only a slight dependence on the density profile of the ejecta is present.

Another point that we want to emphasize is that, as shown by Shivamoggi (1982), a strong magnetic field might lead to larger values of the critical density. A very weak wind magnetization is sufficient to make the interface stable, even weaker than what is usually assumed, based on the dynamical constraints of the nebular evolution (Kennel \& Coroniti 1984). On the other hand, due to the steep internal gradient, the effective value of the shell density in our problem is likely higher than $\rho_{\text {sh }}$ (Eq. (6)).

With regard to the time evolution of the system, as pointed out by Bucciantini et al. (2004), if the effect of pulsar spindown is included, for times greater than the spin-down characteristic time, the expansion tends asymptotically to a linear behavior $R_{\text {pwn }} \propto t$. This means that the local acceleration will drop to zero and the RT instability will be suppressed at later times more efficiently than in the constant pulsar luminosity case.

One of the major differences between our problem and the classical description is that the latter assumes a time independent background condition and does not take into account dynamical effects of the evolution itself. While we do not expect this to have strong effects on the stability (which is an instantaneous condition), if the time scale for the development of the instability is of the same order the time scale of the PWN-SNR system evolution, the growth of the perturbation will be significantly altered.

During the expansion, new, more homogeneous material gathers on the shell. In this case, instead of an exponential growth, we expect that the perturbation will increase as a power law in time (i.e. less efficiently). This is simply seen as follows. From Eq. (1) the instantaneous growth rate in the HD limit is:

$w(t)=\sqrt{\frac{2 \pi \beta(\beta-1)}{\theta}} t^{-1}$,

where we have used the expressions for $g$ and $\rho_{\text {sh }}$ derived above and the fact that the density of the ejecta is much higher than the enthalpy of the PWN. Again, in Eq. (11), there is no dependence on the pulsar luminosity, nor on the energy and mass involved in the SN. Integrating in time, the growth of the perturbation turns out to be given by:

$\exp \int_{t_{0}}^{t} \sqrt{\frac{\beta(\beta-1)}{\theta}} z^{-1} \mathrm{~d} z$

\subsection{Rayleigh-Taylor and Thin-Shell instability}

The interface between the PWN and the shell of swept up ejecta will be in principle subject also to other kinds of instabilities, resulting in a more complex evolution. One of the most important types of instability that may arise in wind bubble systems is the Thin-Shell (TS hereafter) instability. The TS instability has been studied in detail in various environments, from the generic bounded slab problem (Vishniac 1983, 1994) to radiative SNR (Chevalier \& Blondin 1995; Blondin et al. 1998) and planetary nebulae (Carpenter et al. 2001). This instability arises when a thin shell (thickness much smaller than the curvature radius) is forced to bend as a consequence of density perturbation. In the plane parallel case the instability results in the formation of a strongly turbulent mixing layer. However, in spherical geometry, the shell can bend without undergoing disruption (Carpenter et al. 2001). In our case, the TS instability will thus tend to compete with the RT instability for large wavelength perturbations, i.e. perturbations on scales much larger than the relative thickness of the shell $(\theta \gg \pi / 50)$. For smaller scales the RT instability might have the fastest growth. Numerical simulations might help to determine the threshold between the regimes of dominance of either kind of instability.

\section{Numerical simulations}

All the simulations have been performed using the recently developed scheme by Del Zanna et al. (Del Zanna \& Bucciantini 2002; Del Zanna et al. 2003). We refer the reader to the cited papers for a detailed description of the code, and of the equations and algorithms employed. This is a high resolution conservative (shock-capturing) code for 3D-RMHD based on accurate third order reconstruction ENO-type algorithms and on an approximate Riemann solver flux formula (HLL) which does not make use of time-consuming characteristics decomposition. The code employs the Upwind Constrained Transport algorithm (UCT) to ensure the divergence free constraint on the magnetic field to machine accuracy (Londrillo \& Del Zanna 2000; Londrillo \& Del Zanna 2004). The soleinoidal condition is especially important for the present application because we are interested in wave modes parallel to the magnetic field direction.

In principle one should use a different adiabatic coefficient for the PWN $(\Gamma=4 / 3)$ and the SN ejecta $(\Gamma=5 / 3)$, however, in numerical simulations, the use of two different adiabatic coefficients on a contact discontinuity with a very large density jump (density may change by factors of order $10^{5}-10^{6}$ ), leads to the formation of spurious waves that tend to propagate back into the PWN (Shyue 1998; Karni 1998; Kun \& Jishan 1998; Bucciantini et al. 2003). Such spurious oscillations may trigger and artificially amplify the formation of Kelvin-Helmholtz instability at the interface itself, thus 
affecting the stability properties of the system. This is the reason why we have decided to assume a unique adiabatic coefficient, $\Gamma=4 / 3$, both for the PWN and the SNR. It should be noticed that this choice of adiabatic coefficient allows us to reproduce the correct internal structure of the PWN and, as previously discussed (Bucciantini et al. 2003), leads to a value of the total pressure at the boundary that is independent on the wind magnetization. As a consequence the time-evolution of the contact discontinuity and hence the acceleration at the origin of the RT instability turns out to be independent on the wind magnetization. As a side effect, this allows a direct comparison between the hydro and MHD simulations.

Previous simulations by $\mathrm{J} 98$, in the hydrodynamical regime, have been performed using a value $\Gamma=5 / 3$. When compared to those, our simulations lead to a slightly more compressed shell, but we have verified by means of classical HD simulations that there is no significant difference in the growth rates of the instability.

For the same reason we have used second order reconstruction with "monotonized centered" limiter. We have verified that higher order reconstruction, or the use of more compressive limiters, such as "superbee", turns out to enhance the numerical noise at the interface, above the level that can be dissipated by numerical diffusion. The signature of this effect is an excess of secondary $\mathrm{KH}$ features. We want to point out that the problems related to the use of sharper reconstruction are much more severe in our case than in non-relativistic cases, where the density jump is actually reduced by an amount of order the Lorentz factor of the wind.

\subsection{Initial conditions}

The task of following the evolution of PWN-SNR system from few years after the SN explosion up to an age of 2000-3000 years (when the interaction of the PWN with the reverse shock in the ejecta is supposed to happen) requires a huge computational grid and turns out to be extremely time consuming. Moreover, the CFT algorithm employed to guarantee the divergence free condition for the magnetic field is not easily implementable together with Lagrangian remapping (as, for example, in Blondin et al. 2001; Wolfgang \& Blondin 1997), and, on the other hand, implementation of a moving grid is not a trivial task in a proper relativistic contest (Lorentz transformations instead of Galilean).

The reason why these simulations are very time-demanding is that there are two very different time-scales involved in the problem: the evolution time-scale for the PWN, which is set by the velocity of the ejecta, and the integration time-step, which, on the other hand, is determined by the speed of the wind $(c)$. In order to reduce the computational time one may artificially make these two velocities closer than they would be in reality, taking particularly fast ejecta or a slower wind. We reduced the ratio between these two speeds by a factor of 10, gaining a factor of 30 in computational time, although still keeping all the relativistic effects associated with a high Lorentz factor wind.

We have chosen to evolve the system on a $2 \mathrm{D}$ spherical uniform grid $(1500 \times 125$ cells in the $r-\phi$ plane $)$ corresponding to an equatorial section, extending in radius from $0.2 \mathrm{Ul}$ to 21.5 Ul. Simulations have been carried out for different angular sectors from $\pi / 32$ to $\pi / 6$. The radial resolution has been chosen so as to have the shell resolved on at least 20 cells. Concerning the number of cells in the angular direction we have verified that having 125 cells is a good compromise between the need for high resolution and efficiency. At lower resolution numerical diffusion is found to damp the formation of secondary $\mathrm{KH}$ although the growth rate of the RT instability is not affected. Concerning the unit length Ul, it may be useful to mention its value in physical units for a typical PWN-SNR system. For a pulsar wind luminosity (assumed to be constant in time) of $10^{40} \mathrm{erg} / \mathrm{s}$ and an energy release in the SN explosion of $10^{51} \mathrm{erg}$ associated with $3 M_{\odot}$ of ejecta one has $\mathrm{Ul}=1 \mathrm{ly}$. However, we want to emphasize that if the evolution can be approximated as self-similar the properties of RT instability (stability criterion and growth rate) for a given angular perturbation will be independent of the specific choice.

No magnetic field is assumed in the SNR and no radiative cooling is included. An ultrarelativistic wind with Lorentz factor $\gamma=100, p / \rho c^{2}=0.01$ and toroidal magnetic field is injected at the inner radius. Continuous conditions (0th order extrapolation) are used at the outer boundary. Periodic conditions are imposed in the angular direction.

Initial conditions at time $t_{0}$ (corresponding for the above described system to an age of 200 years after the SN) are derived using a much higher resolution $1 \mathrm{D}$ simulation starting from a time $t \ll t_{0}$ after the SN explosion. This choice allows us to start from a situation in which the system is almost completely relaxed on the self-similar solution. No transient phase is observed, contrary to the results in J98. The evolution is followed up to an age of about $10 t_{0}$. An initial perturbation is imposed on the swept up shell density:

$\rho_{\text {sh }}=\rho_{\text {sh,unpert }}+\Delta \rho \cos (2 \pi \phi / \theta)$

where $\phi$ is the angular coordinate, $\theta$ is the angular scale of the perturbation and $\Delta \rho$ is the initial amplitude of the density perturbation. All the simulations consider just a single wavelength. To cut down computational time no multimode case has been studied. No perturbation is assumed in the free expanding ejecta.

Different values of the magnetization parameter $\sigma$ (with $\sigma$ the ratio between magnetic and total luminosity of the wind) have been used. In the standard one-dimensional PWN theory (Rees \& Gunn 1974; Kennel \& Coroniti 1984) the value of the magnetic field at the contact discontinuity is determined by the magnetization of the wind and by the nebular expansion velocity. Having chosen to increase by a factor of 10 the ratio between the latter and the velocity of the wind with respect to a typical case, a magnetization correspondingly higher than the usually mentioned values is required to achieve equipartition at the boundary.

Larger than usually mentioned values of $\sigma$ are required to achieve equipartition at the boundary. In our settings a wind with $\sigma \simeq 0.03$ will result in equipartition between magnetic and thermal pressure at the boundary.

Simulations have been carried out also for lower values of $\sigma$ (namely $\sigma=0.01,0.005,0.0025$ ) which result in a 
pressure dominated interface. Comparison between simulations with different magnetization is aimed at estimating how the development of the instability is affected by the presence of a parallel magnetic field, and, in particular, whether in a magnetic dominated regime, magnetic compression is sufficient to stabilize the system.

\section{Results}

\subsection{Hydrodynamical simulations}

We start our investigation of the development of RT instability by considering the hydrodynamical regime. This is a good approximation when the tension and pressure of the magnetic field are not important for the dynamics of the fluid at the interface. Previous work by J98 was aimed at a study of the global evolution of the PWN-SNR system under the effect of the multimode perturbation originating from numerical noise. We will here focus instead on the development of monochromatic perturbations of different angular extent, analyzing their effects on the shape of the shell in the relativistic regime.

The HD simulations where carried out for perturbation of angular sizes: $\pi / 6, \pi / 8, \pi / 12, \pi / 16, \pi / 24, \pi / 32$. In all the simulations whose results are here presented the amplitude of the initial density perturbation was taken to be $\Delta \rho / \rho=0.25$. The choice of such a large amplitude of the initial perturbation was motivated by computational reasons (we wanted the finger to extend on a large number of cells of the fixed computational domain in a reasonable time). However we checked that the adopted value of $\Delta \rho / \rho$ was still small enough not to affect the growth-rate of the fingers (we have checked that starting with a perturbation 0.1 we end up with a finger that is about one half shorter).

Looking at Fig. 3, initially we see a superlinear phase (see also Jun et al. 1995). The large amplitude assumed for the perturbation, together with the fact that the initial unperturbed state of the system was derived from a much finer grid, are at the origin of this transient, lasting for a time $t \lesssim t_{0}$. At later times, the interface enters a linear growth phase, during which it bends to form a bump into the lighter fluid. Finally a non-linear phase begins, when secondary KH-type instabilities at the head of the finger result in the formation of a mushroom cap.

In Fig. 1 we can notice a number of differences in the simulation results corresponding to initial perturbations of different angular size. Large scale perturbations $(\pi / 6, \pi / 8)$ lead to a uniform distortion of the whole shell. This behavior is more typical of the TS rather than RT instability. When the former is the main process at work, the shell material is not efficiently advected toward the bump, and the density contrast between the wings and the center of the interface distortion keeps almost constant during the evolution.

There is a transition between the regime of dominance of RT versus TS instability at wavelengths corresponding to $\theta \sim \pi / 8$. For this value of $\theta$ the whole shell still shows a bending typical of the TS but a rather elongated finger, likely related to an intervening contribution of RT instability, is now present at its bottom. This behavior can also be appreciated looking at
Fig. 5 where the power spectrum of the normalized density distribution is shown. For $\theta=\pi / 8$ we see that the power is still on the scale of the original perturbation, while we start to observe a decay to smaller scales, associated to the formation of a narrow finger only for $\theta \gtrsim \pi / 12$.

As expected the growth of the finger is more rapid at larger wave numbers and it seems to converge for small scale perturbations. This is probably the effect of the intervening $\mathrm{KH}$ instability that drags away material from the head of the finger: this effect naturally occurs earlier at smaller scales.

Another point to notice is that no secondary KH-type instability along the finger is actually present in simulations with $\theta>\pi / 16$. This is due to the combination of two main effects: first of all, the larger growth rate of RT instability at smaller wavelengths causes a larger shear along the boundaries of the finger providing a more effective trigger for the $\mathrm{KH}$ instability; moreover, as the resolution in angle improves, numerical noise, that may act as a seed for the instability is dissipated less efficiently.

Our simulations show that the formation of mushroom caps on top of the fingers is a quasi-periodic process. When a mushroom cap is formed its typical density is about 2 orders of magnitude below that in the head of the finger, and the drag exerted on its wing by the lighter fluid can force it to shift toward the base where it collides with the swept up shell of ejecta. In the meanwhile, shear on the head causes the formation of a new mushroom cap and the process starts again. We notice also that in none of our cases the finger penetrates the free flowing wind region and the thickness of the mixing layer extends in the case $\theta=\pi / 32$ for about one quarter of the size of the entire nebula. As the head of the finger recedes toward the termination shock, the velocity of the relativistic fluid in front of it increases and so do the drag and the shear thus reducing the growth itself. This result is in principle related to the initial perturbations. However the perturbation we adopted is fairly high, and even extrapolating to later times in the evolution it would take $\sim 40 t_{0}$ to penetrate the termination shock. Given that the free expansion phase lasts for about 3000-4000 years this means that, even neglecting the high shear the head of the finger would be subject when moving to the inner part of the nebula, the instability needs to start since $50 \mathrm{yr}$ after the $\mathrm{SN}$ in order to affect the relativistic wind at very late times (spin-down will cause the termination shock to recede even more toward the pulsar, Bucciantini et al. 2004), and it is not clear if at such an early time the PWN has already set into self-similar expansion. So we deem this result can be generalized as a common property of the filamentary network in PWNe.

The efficiency with which material is actually forced to converge into the finger strongly depends on the size of the initial perturbation. The less the mass that moves from the shell into the finger, the less the shell deformation will be: in the case of shortest wavelengths both the surface density and the total mass in the shell are actually less than in the unperturbed case, causing a slightly larger nebular radius. Looking at Table 1 we see that in the HD case the mass in the mixing layer (finger plus turbulent $\mathrm{KH}$ structure) ranges from 2 to 4 times the mass in the shell: a result in agreement with previous simulations in classical HD regime by J98. However we want to stress that 

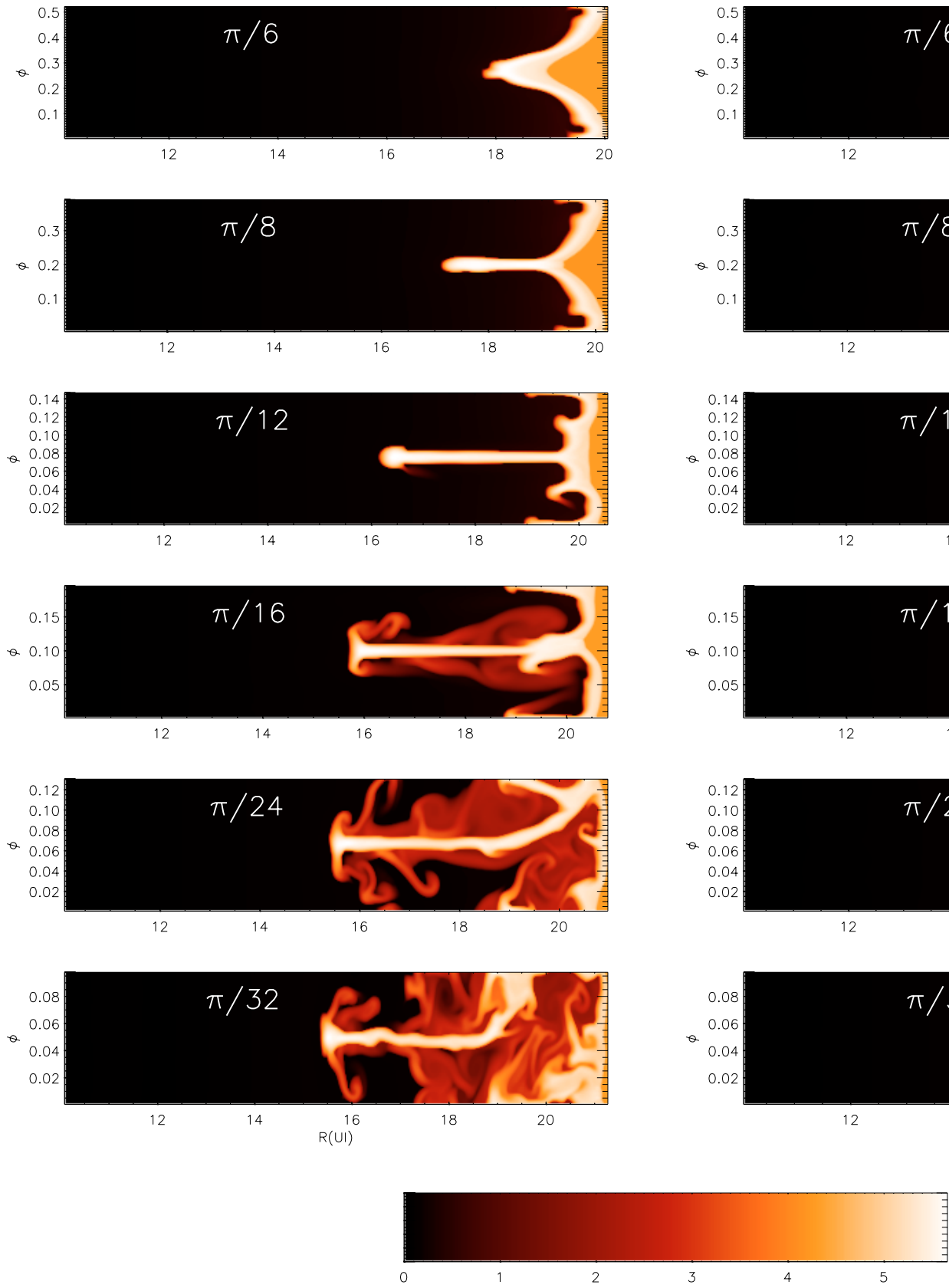

Fig. 1. Structure of the RT unstable interface for different angular scales of the initial perturbation (logarithmic color-scale plot of the density) at $t=10 t_{0}$. The panels on the left refer to the case $\sigma=0$; those on the right to the case $\sigma=0.0025$. Notice that in the magnetized case the efficiency of RT is maximum for angular scales of the initial perturbation between $\pi / 12$ and $\pi / 16$.

even a large change in the total mass of the shell implies only minor changes in the evolution of the PWN size. In the case when $\theta=\pi / 32$, despite $80 \%$ of the total swept-up mass is in the mixing layer (that, as we mentioned, extends for about one quarter of the size of the nebula) the final radius of the PWN is only $10 \%$ larger.

From the point of view of observations, it may be interesting to notice the following. When fingers are well developed, the density in the head can be 2-3 times higher than the maximum value reached in the shell. Therefore, if one expects radiative cooling to be effective in the shell, it will be much more so in these clumps, where the gas may be only partially ionized.
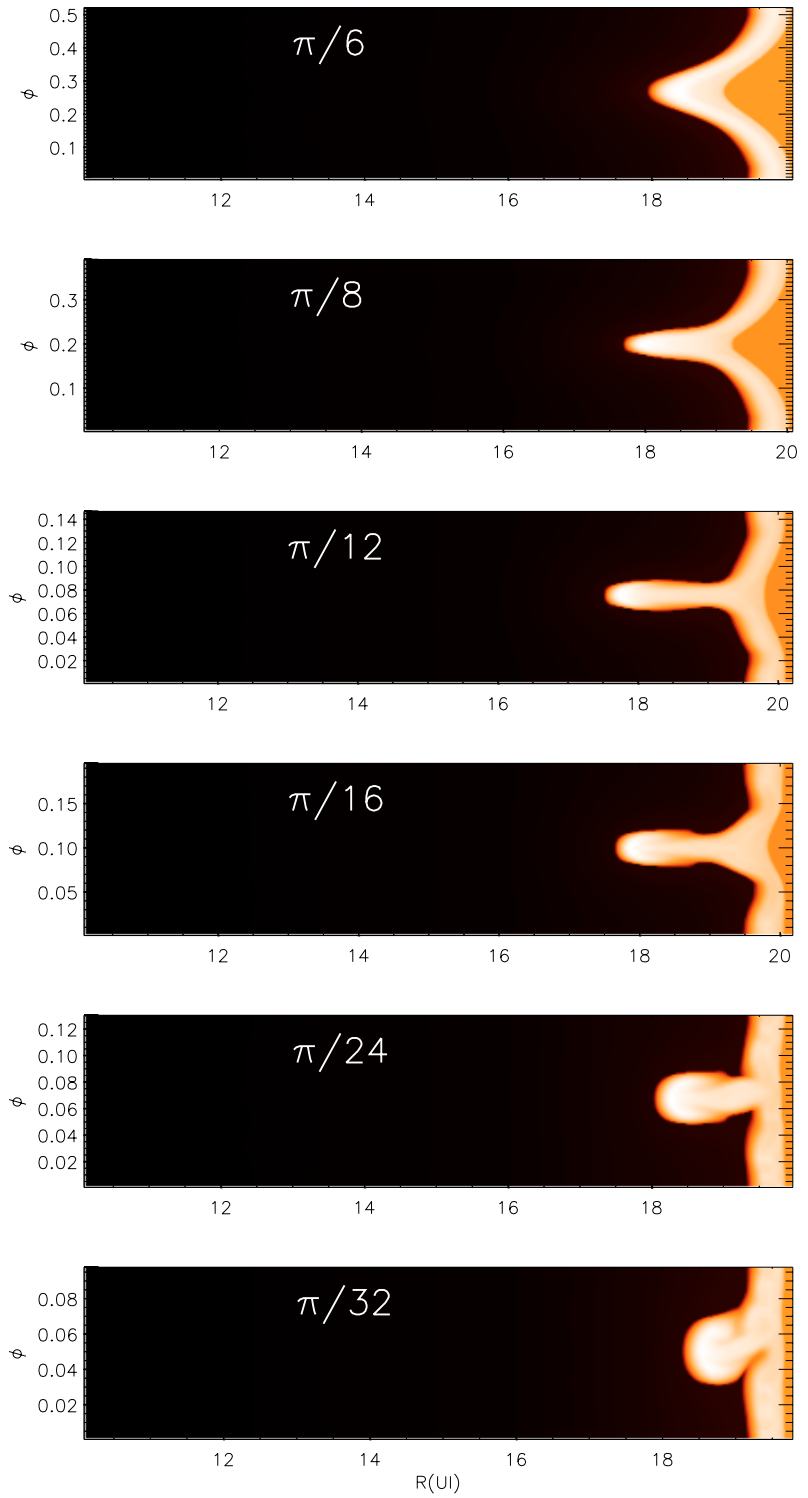
Table 1. Ratio between the mass in the finger and the mass in the shell at $T=5 t_{0}$ and $T=10 t_{0}$.

\begin{tabular}{lllllll}
\hline \hline$T=5 t_{0}$ & & \multicolumn{7}{l}{} \\
\hline$\sigma$ & $\pi / 6$ & $\pi / 8$ & $\pi / 12$ & $\pi / 16$ & $\pi / 24$ & $\pi / 32$ \\
\hline 0.0 & 0.10 & 0.25 & 0.60 & 0.70 & 1.30 & 2.00 \\
0.0025 & 0.05 & 0.15 & 0.45 & 0.60 & 0.75 & 0.75 \\
0.005 & - & 0.10 & - & 0.50 & - & 0.60 \\
0.01 & - & 0.05 & - & 0.30 & - & 0.35 \\
0.03 & - & 0.00 & - & 0.00 & - & - \\
\hline$T=10 t_{0}$ & & & & & & \\
\hline$\sigma$ & $\pi / 6$ & $\pi / 8$ & $\pi / 12$ & $\pi / 16$ & $\pi / 24$ & $\pi / 32$ \\
\hline 0.0 & 0.30 & 0.55 & 1.20 & 1.80 & 3.50 & 4.20 \\
0.0025 & 0.25 & 0.40 & 0.70 & 0.75 & 0.80 & 0.75 \\
0.005 & - & 0.40 & - & 0.75 & - & 0.70 \\
0.01 & - & 0.20 & - & 0.60 & - & 0.35 \\
0.03 & - & 0.00 & - & 0.00 & - & - \\
\hline
\end{tabular}

disruption while in the former cases the finger even if shorter is still straight and attached to the shell.

As noticed by Wang \& Nepveu (1983) another interesting aspect of RT instability is the complementary behavior of the heavy and light fluids: "as one falls in a given sector of the boundary, inducing the other to rise in other sectors of it, large scale vortex motions are set up which result in the formation of interlocking mushroom structures". This is exactly what we observe at the boundary of the simulation box in $\phi$. Large circulatory motions produced by the development of the primary RT fingers at the center of the box, coupled with secondary $\mathrm{KH}$ instability give rise to the formation of smaller fingers at the boundary itself. These fingers are present even in simulations carried out with a lower resolution, where mushroom cap formation is damped by numerical diffusion.

Looking at the turbulent structure in the mixing layer we observe a rapid growth of the curl of the velocity field once the secondary $\mathrm{KH}$ starts to create caps and vortexes along the finger. Once the mixing layer is well developed the vorticity seems to saturate and its growth is strongly reduced.

In all cases where RT is the dominant instability $(\theta \geq \pi / 8)$, the thickness of the finger progressively decreases until it is only of order one tenth of the size of the initial perturbation. This can be largely attributed to the ram pressure exerted by the surrounding relativistic fluid, as it moves around in a circulatory pattern. Again this can be seen looking at Fig. 5 where the width of the power spectrum of the density distribution is $\sim 10$ in unity of the wave number of the original perturbation.

As far as the power spectrum of the mass distribution is concerned, we found it useful to introduce the radial column density $\eta$ as the quantity to look at. We defined $\eta$ as:

$\eta(\phi)=\int_{\mathcal{L}} r^{2} \rho(r, \phi) \mathrm{d} r$

where $\mathcal{L}$ is the radial extent of the mixing layer (from the front shock in the swept up shell to the head of the finger). In Fig. 5 we show the power spectrum of $\eta(\phi)$. We see from the figure that, except for the case $\theta=\pi / 6$ where almost all the power is still at the scale of the original perturbation, in all other cases there is evidence for the formation of structures on scales as small as $\sim 1 / 20 \theta$.

We want to point out that we decided to trace the growth of the fingers by measuring their length. With respect to Jun et al. (1995), where the growth rate is measured by using the amplitude of the density perturbation, one might expect the comparison not to be straightforward. Our choice was motivated by the need to provide a growth rate by means of parameters (the length of the finger) that can in principle be easily observed, while Jun et al. (1995) choice was probably dictated by the need of a comparison with theory (in our case there is no theory for comparison). However if one follow the evolution of the density perturbation (Table 1) we find that the standard behaviors of Fig. 3 (like saturation and magnetic compression) is retrieved.

\subsection{Magnetohydrodynamical simulations}

The widely accepted picture for PWNe leads to believe that at the contact discontinuity with the SNR the relativistic bubble should be magnetically dominated. Both the one dimensional models by Kennel \& Coroniti (1984), and Emmering \& Chevalier (1987), as well as the 2D stationary model by Begelmann \& Li (1992), give a ratio between the magnetic and the thermal pressure at the boundary of the nebula close or above equipartition. To investigate how the magnetic field affects the development of the RT instability in a representative regime, the pulsar wind magnetization has to be chosen so as to guarantee the proper conditions at the interface. Given our velocity normalization, as well as the PWN-SNR evolution, we have determined that a pulsar wind with $\sigma=0.03$ results in an equipartition condition at the boundary.

It is well known that the presence of a strong magnetic field can suppress the RT instability. In fact, for $\sigma=0.03$ no finger is formed for any of the initial scales of the perturbations considered: only a negligible deformation of the contact discontinuity is present, in spite of the large density perturbation amplitude (25\%) adopted. 

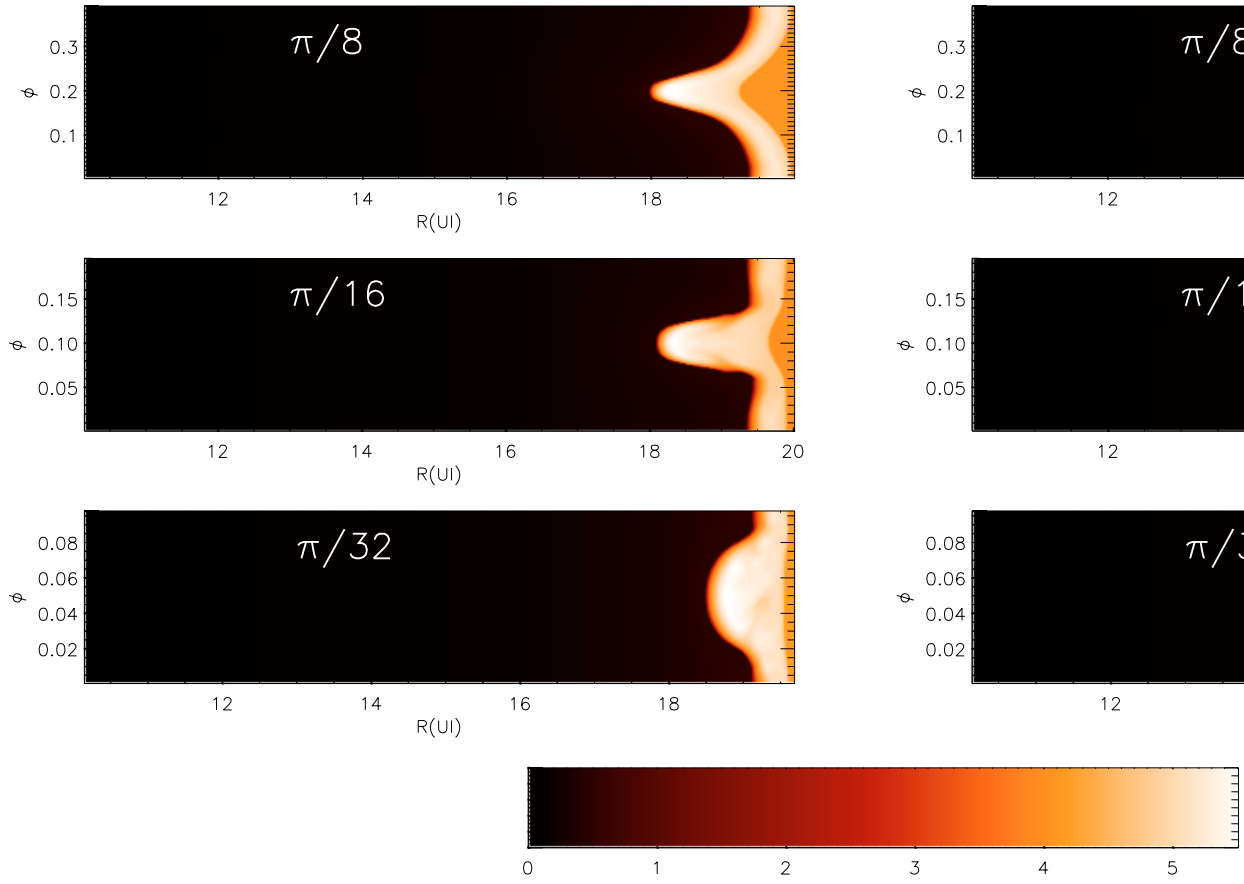

Fig. 2. Same as in Fig. 1 but for higher values of the PWN magnetization. The panels on the left refer to the case $\sigma=0.005$; those on the right to the case $\sigma=0.01$. Now the maximum growth rate for RT has moved to larger angular sizes $\sim \pi / 8$.

To better understand how strong the suppression of RT instability could be, and what modifications are to be expected when the system is unstable, we have also investigated cases of lower wind magnetization: $\sigma=0.01,0.005,0.0025$. This will also clarify the transition between stable and unstable regimes.

The $\sigma=0.01,0.005,0.0025$ cases give a PWN where the ratio between magnetic and thermal pressure at the contact discontinuity is, respectively, $P_{B} / P_{t} \sim 1 / 5,1 / 15,1 / 30$. The system is progressively more stable with increasing magnetization (Figs. 1-3), but even a weak magnetization has important effects on the finger structure and evolution.

A comparison between the case with $\sigma=0.0025$ and the HD simulations shows that:

- The growth rate is strongly reduced. For all the magnetization values we considered, the growth of the finger is maximum for $\theta$ in the range $\pi / 12-\pi / 16$ (Figs. 1 and 3 ).

- Secondary KH instability is completely suppressed. No mushroom cap, nor secondary fingers at the boundary are formed, and the density profile of the shell is not much affected. In the magnetic case there is no turbulent mixing layer and the CD is well defined. There is no increase in the curl of the velocity field, at variance with the correspondent turbulent HD cases. The flow is still laminar.

- The fingers tend to be thicker and do not show fragmentation. The cascade to small scales is much less efficient than in the HD case, as it is clear from the power spectrum of $\eta$ (Fig. 6). The density in the head of the finger is about a factor 2-4 times higher than the maximum in the shell. This ratio tends to be slightly higher than in the corresponding HD case: this is related with the fact that now the finger is more compact (see also the presence of a secondary bump in the power spectrum in Fig. 6).
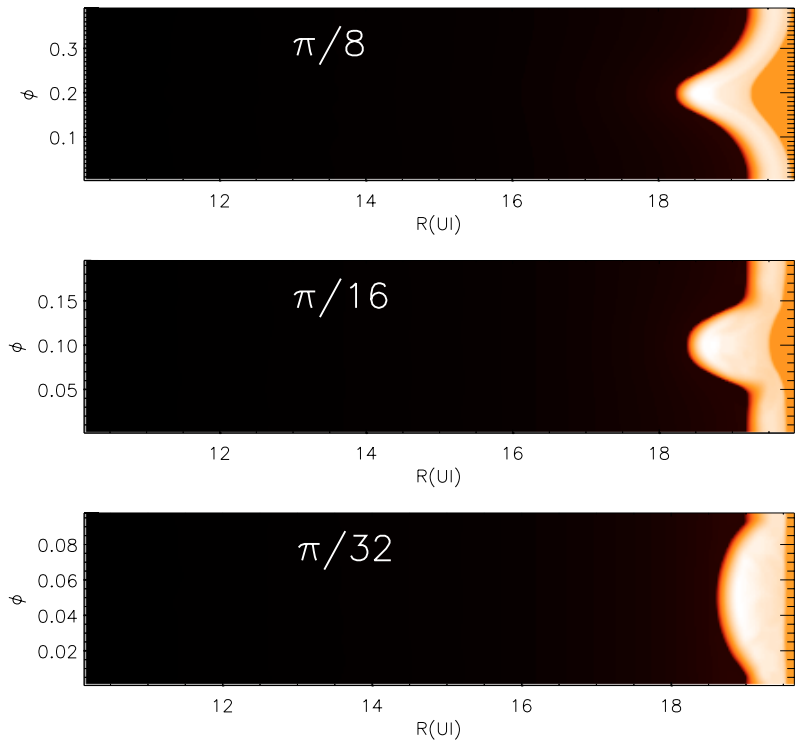

- There is a maximum length the finger can achieve, that depends on the wavelength and on the magnetization. We found that once the magnetic field compressed in front of the finger reaches values close to equipartition, the growth stops and in some cases the length of the finger is even slightly reduced (Fig. 3).

- In all cases when a finger is formed, the magnetic field lines are bent. Depending on $\sigma$, the radial component of the field that arises can become comparable (in the $\sigma=0.01$ ) and/or greater $(\sigma=0.0025)$ than the toroidal component.

As the magnetization increases, for small values of $\theta$ (typically $\theta=\pi / 32$ in our simulations) a bump of angular size close to that of the original perturbation is formed, rather than a narrow finger. The evolution of the nebular radius is not much affected because not enough mass is dragged into the finger (see Table 1) and the shell thickness is almost constant during the evolution. Looking at Fig. 6 we can seen the same behavior from the density distribution. Again we notice that a tail to small scales is developed only for the cases $\theta=\pi / 8-\pi / 16$, where the growth of the finger has not stopped yet (Fig. 3), while it is suppressed at smaller scales due to magnetic compression. We also notice that the finger tend to be thicker than in the analogous HD case. Despite the morphological difference between the cases $\theta=\pi / 32$ and $\theta=\pi / 6$ the density distribution is fairly similar apart for the presence of secondary bumps that are indicative of the presence of a finger with a flat density distribution. Simulations are able to reproduce the density gradient inside the swept-up shell of ejecta and the local density at the interface is about one order of magnitude higher than the average value that we use in Eq. (10). In principle one might expect the effective density to be higher at smaller scales (where the average should be computed on a scale of order $\lambda$ 


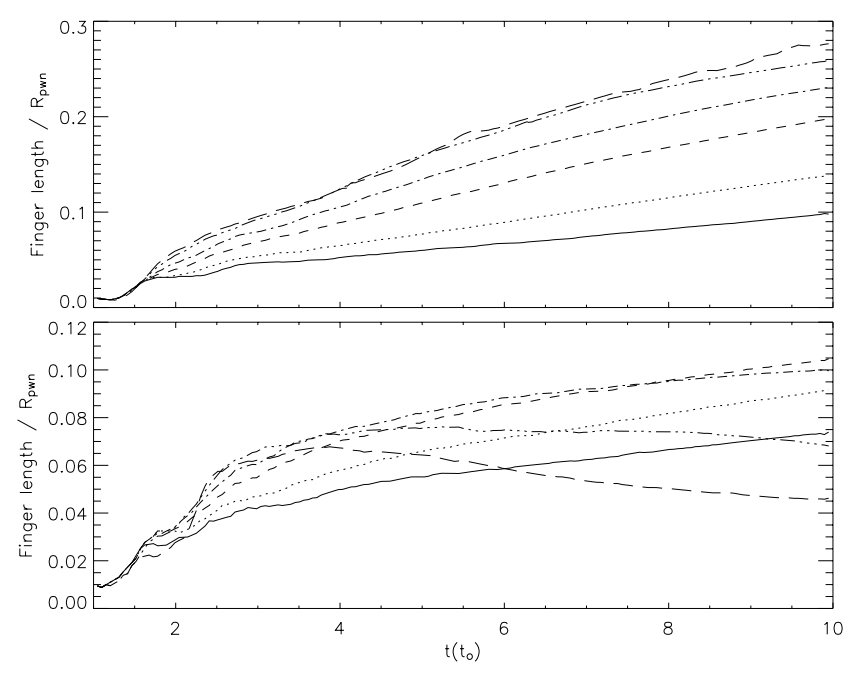

Fig. 3. Evolution of the ratio between the length of the finger (radial extent of the mixing layer in the turbulent HD case) and the radius of the PWN $R_{\mathrm{cd}}$. The various curves refer to different initial perturbations: solid $\theta=\pi / 6$; dotted $\theta=\pi / 8$; dashed $\theta=\pi / 12$; dot-dashed $\theta=\pi / 16$; dash-triple-dot $\theta=\pi / 24$; long-dashed $\theta=\pi / 32$. The upper panel refers to the case $\sigma=0$; the bottom panel to $\sigma=0.0025$. After a superlinear phase, lasting for about $0.5 t_{0}$ for all values of $\theta$, we observe a linear phase that for small $\theta$ in the HD regime last up to about $t \sim 6 t_{0}$, while in the MHD cases it stops earlier at around $t=\sim 4 t_{0}$. The growth of the finger saturates at later times for the smallest wavelengths. See text for a detailed description.

rather than on the entire thickness of the shell), hence satisfying more easily the requirements for instability. Despite this, when a magnetic field at equipartition is present, even in the case $\theta=\pi / 6$, which in principle should be below the threshold value $(\theta \sim \pi / 10)$ for stability provided by Eq. (10), no deformation is observed (neither a relaxation to the imposed initial perturbation). Given the simplified assumptions used to derive the threshold value itself, like constant density or steady state background, and the reduction to 2 dimensions of our numerical simulations we are not able to specify the reason for such results. It well might be an effect of a strong magnetic field, as anticipated is Sect. 2.1. Introducing the correction provided by Shivamoggi (1982) the threshold value should decrease to $\theta \sim \pi / 5$, in agreement with the numerical results. In the case of a magnetic field five times below the equipartition value, we find that a fingers forms for all the values of $\theta$ we investigated, in agreement with prediction from Eq. (10). However even in the cases where a finger develops its growth seems to saturate when the field in the head is compressed to equipartition value thus suggesting the presence of some stabilizing effect that the standard theory does not account for (further investigation is under development). It is interesting to notice that even if the interface does not bent at all, the density perturbation in the swept-up shell does not disappear: the relative amplitude is obviously reduced as a consequence of new material gathering on the shell, but the original perturbation is frozen. This confirm that stability does not change with time as the evolution proceeds. This result shows the limitations of former simulations in pressure dominated regimes where the standard stability criterion was found to give good results. Nonetheless,

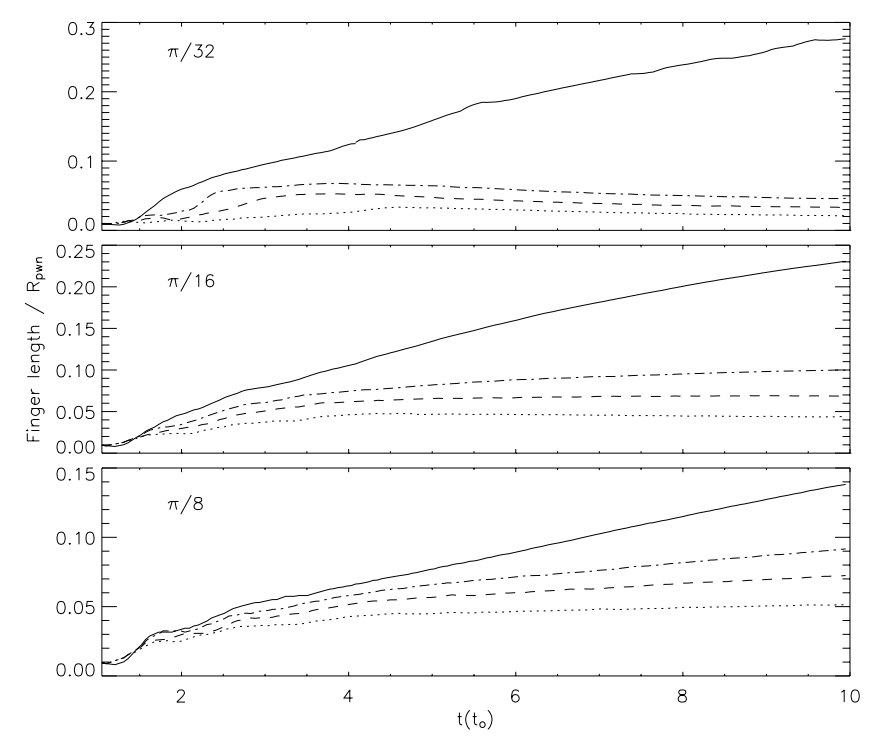

Fig. 4. Evolution of the ratio between the length of the finger and the radius of the PWN $R_{\mathrm{cd}}$. The upper panel refers to $\theta=\pi / 32$; the middle panel to $\theta=\pi / 16$; the bottom one to $\theta=\pi / 8$. Different curves refer to different values of the wind magnetization: solid $\sigma=0$; dotted $\sigma=0.001 ;$ dot-dashed $\sigma=0.0025$.

other results concerning the shape of the perturbed interface, the formation of secondary KH instability, as well as the existence of a maximum deformation, still hold (Jun et al. 1995).

Our results seem to agree with those of Wang \& Nepveu (1983) in the context of accretion onto a compact object. The actual threshold values derived from Eq. (10) seems to be underestimated, but further analysis to clarify this point is required.

\subsection{Implications of the MHD results}

Our results show that once the magnetic field is included in the evolution of the PWN inside its SNR the statement that the accelerated shell of ejecta should be RT unstable is not so trivial as it has been considered. As we have already stressed, since the acceleration is basically independent of the exact value of the magnetic field, but depends only on the pulsar total luminosity and SNR properties, a magnetic field above or close to equipartition can suppress the growth of the perturbations parallel to its direction, even of the large scale ones.

This has important consequences on the correct understanding of the physical conditions both at the boundary of the PWN and inside the shell of ejecta. In the case of the Crab Nebula, H96 concluded that the shell must be subject to efficient cooling. Using the standard values (Chandrasekhar 1961) for the critical threshold, H96 finds that the shell must have a density at least one order of magnitude greater than the upper limit estimated from the non-detection of the halo (Sankrit \& Hester 1997) assuming adiabaticity. However, the radiative cooling hypothesis is not without problems. Even though the metallicity could in principle be high enough to give the required cooling efficiency, the effectiveness of the cooling process could be partially reduced by the presence of a synchrotron emitting nebula, which can provide 

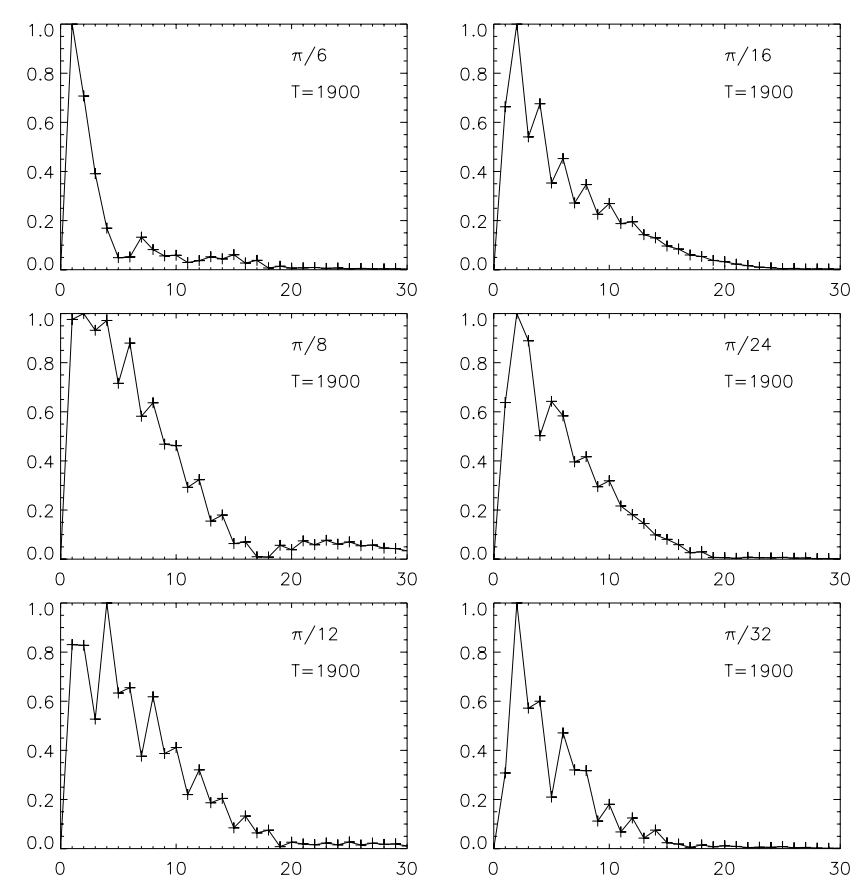

Fig. 5. Spatial Fourier transform of the column density $\eta$ in the region between the head of the finger and the shell of swept-up ejecta for the case $\sigma=0$. The transform is taken at a time $t=10 t_{0}$ and the average has been subtracted. The different panels correspond to different angular sizes $\theta$ of the initial perturbation. The horizontal scale is in units of $1 / \theta$. Except for the case $\theta=\pi / 6$ we observe a cascade to small scales associated with the formation of a narrow finger and structure associated to $\mathrm{KH}$ instability.

enough UV radiation to keep the material inside the shell from cooling. Moreover efficient cooling means that the relative thickness of the shell should be reduced by the same factor by which the density is increased. A much thinner shell would be subject to more efficient TS instability and the threshold between TS and RT would eventually move to smaller scales. As we noticed, in our simulations this threshold falls in the range $\theta \sim \pi / 6-\pi / 8$. If the cooling reduces the thickness by a factor of 10 we might expect the threshold angular size to drop by about the same factor. This means that the observed size (H96) of the perturbations in the Crab Nebula would be close to this threshold.

If cooling is required as a key ingredient for instability in PWN-SNR systems, it is highly probable that the efficiency of finger formation was higher during the early stages of the evolution. The cooling efficiency, in fact, strongly depends on the density of the shell, which is expected to have been much higher in the early phases. The system could have experienced a radiative cooling phase at the beginning leading to the development of RT fingers. In the following evolutionary phases, it could have become non-radiative, due to the decrease in the shell density. In the case of the Crab Nebula, for example, the general consensus is that this young system is close to the above mentioned transition, although there is evidence that cooling might still be going on (Graham et al. 1990).

Another possible explanation for the origin of the filamentary network is that it is caused by dense clumps formed during the SN explosion. Clumps are currently observed in the
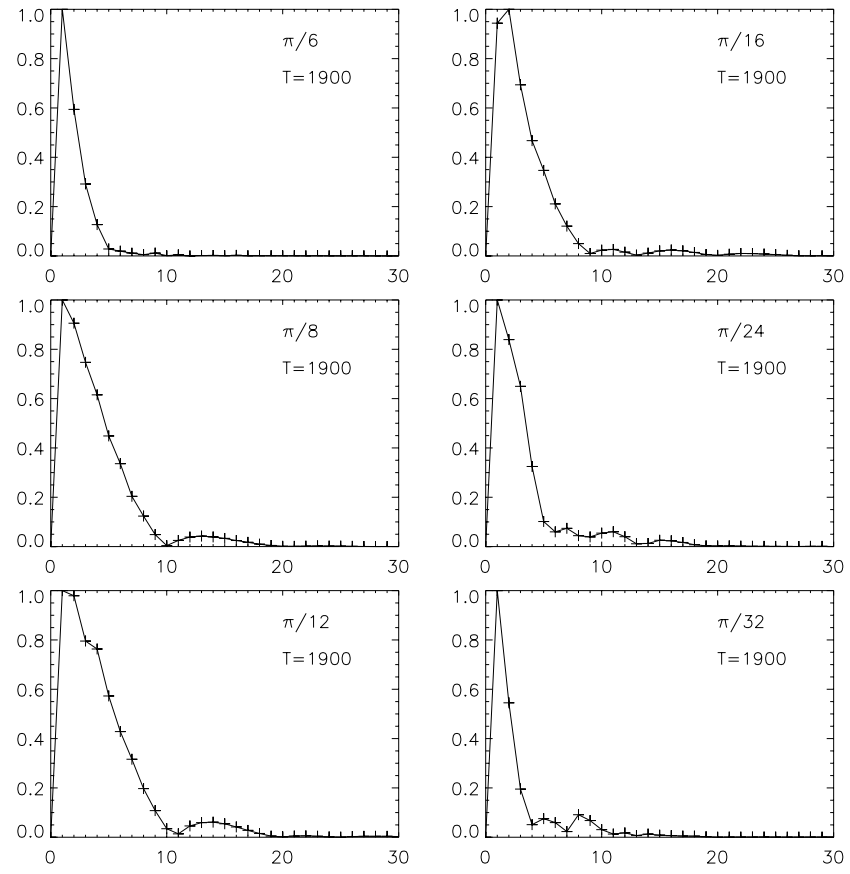

Fig. 6. Same as Fig. 5 but for the case $\sigma=0.0025$. We notice that now a bump appears in the power spectrum in most panels at around $1 / 10-1 / 20 \theta$. This is associated to the formation of a thick finger.

remnant of SN1987A (Wang et al. 1996). In this scenario, rather than to a local interface instability, the fingers would be due to the inertia (or buoyancy) of these dense clumps, which have resisted the PWN expansion. However our lack of knowledge of the typical SNR clumpiness as well as our ability to measure the density in the fingers do not allow us to confirm or to rule out this hypothesis.

We must point out that the standard picture of a PWN with magnetic pressure close to equipartition at the boundary comes from theoretical models that assume the plasma flow to be laminar from the termination shock to the contact discontinuity. More recent developments in this field suggest that the flow structure may be rather complex and, even for wind magnetizations such that the average nebular field is around equipartition, the ratio between magnetic and thermal pressure at the boundary may strongly depend on latitude (Bogovalov \& Khangoulian 2002; Lyubarsky 2002; Komissarov \& Lyubarsky 2003; Del Zanna et al. 2004) and be below equipartition in some regions.

\subsection{Limits of the model}

In this section we want to discuss some limitations intrinsic of our approach. Apart from the absence of cooling that could provide a key ingredient in the correct modeling of the proper physical conditions at the interface, the major limitation is the reduction of degrees of freedom of the system with respect to a proper 3D case. Unfortunately, proper 3D simulations are prohibitive in terms of computational time, even if simplified plane parallel cases in magnetic dominated environments surely deserve more attention. In addition to all of this, we would also like to remind the reader that our investigation was performed 
only for single wavelength modes, thus ignoring all the possible consequences of mode coupling.

In $2 \mathrm{D}$ the magnetic field in the head of the finger can be compressed very efficiently leading to high magnetic pressure and damping the growth of the instability. In a more realistic 3D situation the magnetic field lines are also allowed to move in the transverse direction and to slide around the finger. This will eventually reduce the compression in the head so that well formed fingers can actually protrude much further in the relativistic bubble. This effect will also lead to a deformation of the fingers into sheet like structures.

\section{Conclusion}

In this paper, for the fist time, we present simulations of RT instability for PWNe expanding into the SNR ejecta, including the effect of a magnetic field at the boundary. Simulations were carried out for various initial angular perturbations. In the HD regime our simulations confirm previous results by $\mathrm{J} 98$ concerning the nebular size and fraction of mass contained in the mixing layer. The higher resolution we adopted allows us to follow the detailed evolution of the finger structure. We find that finger fragmentation is likely to happen only once a well developed turbulent mixing layer has formed, and that, for large scale perturbations, TS instability seems to dominate over RT, leading to an overall deformation of the swept-up shell of ejecta. We were able to observe the dragging exerted by secondary $\mathrm{KH}$ and the formation of mushroom caps. From our simulations it seems that fingers do not grow enough to penetrate the relativistic wind region and the mixing layer extends to about one quarter of the nebular radius.

The introduction of a magnetic field turns out to be a key ingredient for a correct understanding and modeling of the RT instability. We have shown that the stability criterion adapted to the self-similar PWN-SNR evolution, does not depend on pulsar wind luminosity, SN mass and energy and does not change during the nebula evolution. The result is that the ratio between the shell density and the critical density is close to unity if a magnetic field around equipartition is assumed at the PWN boundary. This result is confirmed by our simulations that show that magnetic field close or above equipartition can completely suppress the RT instability even for large scale perturbations. A weaker magnetic field is able to reduce the growth of the finger leading to a round rather than elongated protuberance attached to the ejecta shell. A non-negligible magnetic field can suppress the secondary KH completely: no turbulent mixing layer is formed, and fingers are thicker than in the HD case. This is the main difference with respect to former simulations in the pressure dominated regime, where the standard stability criterion was found to give the correct result.

As shown by $\mathrm{H} 96$ an efficient cooling is required to explain the RT filamentary network observed in the Crab Nebula. Our simulations however point out that this requirement is even stronger than previously thought, thus favoring the hypothesis that the fingers formed during the early phases of the system evolution, when the density of the shell was higher. An alternative to efficient cooling would be the presence of dense clumps formed during the $\mathrm{SN}$ that may resist the expansion of the nebula.

Recent work on axisymmetric pulsar winds seems also to suggest a third possible explanation for the formation of RT fingers and possibly their latitude distribution. If turbulent large scale convective cells are formed, as a consequence of ram pressure gradient in the wind, there might be regions at the boundary where the magnetic field is below equipartition and the RT instability criterion is satisfied. This scenario deserves more attention, however 3D global simulations with high resolution are still too much demanding in terms of computational time.

Acknowledgements. This work has been partially supported by the Italian Ministry of University and Research (MIUR) under grant COFIN2002, by INAF under grant COFIN 2002, and partly by a SciDAC grant from the US Department of Energy High Energy and Nuclear Physics Program. We thank the North Carolina Supercomputing Centre and Oak Ridge National Lab for their generous support of computational resources.

\section{References}

Allen, A. J., \& Hughes, P. A. 1984, MNRAS, 208, 609

Bandiera, R., Pacini, F., \& Salvati, M. 1983, A\&A, 126, 7

Begelman, M. C. 1998, ApJ, 493, 291

Begelman, M. C., \& Li, Z. 1992, ApJ, 397, 187

Blondin, J. M., Lundqvist, P., \& Chevalier, R. A. 1996, ApJ, 472, 257

Blondin, J. M., Wright, E. B., Borkowski, K. J., \& Reynolds, S. P. 1998, ApJ, 500, 342

Bogovalov, S. V., \& Khangoulian, D. V. 2002, Astron. Lett., 28, 373

Bucciantini, N., Blondin, J. M., Del Zanna, L., \& Amato, E. 2003, A\&A, 405, 617

Bucciantini, N., Bandiera, R., Blondin, J. M., Del Zanna, L., \& Amato, E. 2004, A\&A, submitted

Campbell, J. R., Borkowski, K. J., \& Blondin, J. 2003, A\&AS/High Energy Astrophysics Division, 35

Carpenter, A. M., Blondin, J. M., \& Borkowski, K. J. 2001, Am. Astron. Soc. Meet., 199, 136

Chandrasekhar, S. 1961, Hydrodynamic and hydromagnetic stability (Oxford University Press)

Chevalier, R. A., \& Blondin, J. M. 1995, ApJ, 444, 312

Chevalier, R. A., \& Fransson, C. 1992, ApJ, 395, 540

Chevalier, R. A., \& Gull, T. R. 1975, ApJ, 200, 399

Chevalier, R. A., \& Soker, N. 1989, ApJ, 341, 867

Chevalier, R. A., Blondin, J. M., \& Emmering, R. T. 1992, ApJ, 392, 118

Del Zanna, L., \& Bucciantini, N. 2002, A\&A, 390, 1177

Del Zanna, L., Bucciantini, N., \& Londrillo, P. 2003, A\&A, 400, 397

Del Zanna, L., Amato, E., \& Bucciantini, N. 2004, A\&A, submitted

Dwarkadas, V. V., \& Chevalier, R. A. 1998, ApJ, 497, 807

Emmering, R. T., \& Chevalier, R. A. 1987, ApJ, 321, 334, EC

Featherstone, N., Blondin, J., \& Borkowski, K. 2001, Am. Astron. Soc. Meet., 199, 126

Fryxell, B., Arnett, D., \& Mueller, E. 1991, ApJ, 367, 619

Gardner, C. L., Glimm, J., McBryan, O., et al. 1988, Phys. Fluids, 31, 447

Goldreich, P., \& Julian, W. H. 1969, ApJ, 157, 869

Graham, J. R., Wright, G. S., \& Longmore, A. J. 1990, ApJ, 352, 172

Hester, J. J., Stone, J. M., Scowen, P. A., et al. 1996, ApJ, 456, 225, H96

Jun, B. I. 1998, ApJ, 499, 282, J98 
Jun, B. I., Norman, M. L., \& Stone, J. M. 1995, ApJ, 453, 332 Karni, S. 1994, J. Comput. Phys., 112, 31

Kennel, C. F., \& Coroniti, F. V. 1984, ApJ, 283, 694, KC

Komissarov, S. S., Lyubarsky, Y. E. 2003, MNRAS, 344, L93

Kun, X., \& Jishan, H. 1998, J. Comput. Phys., 142, 412

Londrillo, P., \& Del Zanna, L. 2000, ApJ, 530, 508

Londrillo, P., \& Del Zanna, L. 2004, JCP, 195/1, 17

Lyubarsky, Y. E. 2002, MNRAS, 329, L34

Matzner, C. D., \& McKee, C. F. 1999, ApJ, 510, 379

Michel, F. C., \& Li, H. 1999, Phys. Rep., 318, 227

Plesset, M. S., \& Whipple, C. G. 1974, Phys. Fluids, 17, 1

Rees, M. J., \& Gunn, J. E. 1974, MNRAS, 167, 1
Sankrit, R., \& Hester, J. J. 1997, ApJ, 491, 796

Shivamoggi, B. K. 1982, Ap\&SS, 84, 477

Shyue, K. M. 1998, J. Comput. Phys., 142, 208

Stone, J. M., \& Norman, M. L. 1992, ApJ, 390, L17

van der Swaluw, E., Achterberg, A., Gallant, Y. A., \& Tóth, G. 2001, A\&A, 380, 309

Vishniac, E. T. 1983, ApJ, 274, 152

Vishniac, E. T. 1994, ApJ, 428, 186

Wang, Y.-M., \& Nepveu, M. 1983, A\&A, 118, 267

Wang, L., Wheeler, J. C., Krishner, R. P., et al. 1996, ApJ, 466, 998

Wolfgang, C., \& Blonding, J. M. 1997, A\&AS, 29, 787 